\title{
Evaluation of automatic time gain compensated in-vivo ultrasound sequences
}

Axelsen, Martin Christian; Røeboe, Kristian Frostholm; Hemmsen, Martin Christian; Nikolov, Svetoslav Ivanov; Pedersen, Mads Møller; Nielsen, Michael Bachmann; Jensen, Jørgen Arendt

\section{Published in:}

Proceedings of the 2010 IEEE International Ultrasonics Symposium.

Link to article, DOI:

10.1109/ULTSYM.2010.5935929

Publication date:

2010

Document Version

Early version, also known as pre-print

Link back to DTU Orbit

Citation (APA):

Axelsen, M. C., Røeboe, K. F., Hemmsen, M. C., Nikolov, S. I., Pedersen, M. M., Nielsen, M. B., \& Jensen, J. A. (2010). Evaluation of automatic time gain compensated in-vivo ultrasound sequences. In Proceedings of the 2010 IEEE International Ultrasonics Symposium. (pp. 1640-1643). IEEE.

https://doi.org/10.1109/ULTSYM.2010.5935929

\section{General rights}

Copyright and moral rights for the publications made accessible in the public portal are retained by the authors and/or other copyright owners and it is a condition of accessing publications that users recognise and abide by the legal requirements associated with these rights.

- Users may download and print one copy of any publication from the public portal for the purpose of private study or research.

- You may not further distribute the material or use it for any profit-making activity or commercial gain

- You may freely distribute the URL identifying the publication in the public portal 


\title{
Evaluation of automatic time gain compensated in-vivo ultrasound sequences
}

\author{
Martin Christian Axelsen* and Kristian Frostholm Røeboe*, Martin Christian Hemmsen*†, \\ Svetoslav Ivanov Nikolov ${ }^{\dagger}$, Mads Møller Pedersen ${ }^{\ddagger}$, Michael Bachmann Nielsen ${ }^{\ddagger}$ and \\ Jørgen Arendt Jensen* \\ ${ }^{*}$ Center for Fast Ultrasound Imaging, Dept. of Elec. Eng., Bldg. 349, Technical University of Denmark, \\ DK-2800 Kgs. Lyngby, Denmark \\ ${ }^{\dagger}$ BK Medical Aps, Mileparken 34, DK-2730 Herlev, Denmark \\ ${ }_{\ddagger}^{\ddagger}$ Department of Radiology, Copenhagen University Hospital, Rigshospitalet, DK-2100 Copenhagen, Denmark
}

\begin{abstract}
Ultrasound imaging is increasingly being used in applications such as surgery, anesthesia and urology, where the users are not trained radiologists. User studies indicate that these users rarely adjust the controls of the ultrasound scanner. This project presents a preliminary evaluation of a new algorithm for automatic time gain compensation (TGC) on in-vivo ultrasound sequences. Forty ultrasound sequences were recorded from the abdomen of two healthy volunteers. Each sequence of $5 \mathrm{sec}$ was recorded with 40 frames/sec. Post processing each frame, a mask is created wherein anechoic and hyper echoic regions are mapped. Near field hyper intensity and deep areas with low signal strength are also included in the mask. The algorithm uses this mask to create a parallel image where anechoic and hyper echoic regions are eliminated. From this, the mean power is calculated as a function of depth. The power is then used as an estimate of the attenuation, and from this, the needed compensation is found. The measurements were performed by an experienced sonographer using an ultrasound scanner $(2202$ ProFocus, BK Medical, Denmark) with a 192 elements concave transducer (8820e BK Medical). A research interface was used to retrieve unprocessed data from the scanner with no preset TGC, using a standard abdominal setup.
\end{abstract}

Five experts in medical ultrasound evaluated the unprocessed and processed video sequences in a double-blinded randomized trial on image quality and penetration depth. In the evaluation of image quality, the unprocessed and processed sequences were displayed in pairs side-by-side in random order and with random left right placement. Each pair was displayed and scored twice, with different permutations. The sequences were evaluated on their relative clinical value. $P$-values on the order of $10^{-8}-10^{-14}$ indicate that the image quality of the processed sequences are clinically better than the unprocessed. In the evaluation of penetration depth, all the processed and unprocessed sequences were displayed in random order. Each sequence was evaluated on the basis of; at what depth the image quality had decreased so much that it was of no clinical value. The pooled results show a mean increase in penetration depth of $1.91 \mathrm{~cm}$ with a p-value of $1.19 \cdot 10^{-18}$. In conclusion a new algorithm has been developed and evaluated. It is capable of compensating for the depth attenuation on abdominal in-vivo ultrasound images.

\section{INTRODUCTION}

Ultrasound imaging is widely used for several different diagnostic purposes. The fact that ultrasound imaging is a non-invasive, real time imaging modality, makes it easy to approach. However, adjustment of the TGC curve depends on the operator. TGC adjustment is essential for the optimal setting of diagnostic images. It takes time and requires attention from the operator and the TGC settings are difficult to reproduce. Furthermore when the transducer is moved to a different view of the same region or a new focus is set, a post adjustment of the TGC is needed. In practice, these adjustments are rarely performed in the clinical setting. Thus, automatic adjustment of the TGC curve will optimize the image setting and save time for the operator. It will also make it easier for inexperienced users to obtain optimized images. In this study we have implemented a new algorithm for automatic time gain compensation (ATGC) aimed at abdominal scans. Abdominal scans are characterized by large areas of uniform speckle reflections, smaller dark regions due to cysts and blood vessels, and bright reflectors such as the diaphragm. The optimization criteria were to achieve a uniform gray level for the regions characterized by diffuse scattering. As in the work done by Lee et al. [1], the intensity of the image is used as an estimation of the depth attenuation. This estimation is, however, often disturbed by the presence of demarcated structures in the anatomy. These could be cysts, blood vessels, or the diaphragm. In the ultrasound image, they will show as demarcated areas with a deviation in intensity compared to the surrounding tissue. An overview of the imaged anatomy is therefore needed in order to obtain the most accurate estimation of the depth attenuation. The method for mapping and differentiation between different anatomical structures automatically has been inspired by Pye et al. [2,4] and Hughes et al. [5].

\section{Methods And Algorithm}

The performance of the developed algorithm was tested on in-vivo ultrasound sequences acquired through a research interface [3] and these were evaluated by experts.

\section{A. Time Gain Compensation algorithm}

1) Initialization: The developed algorithm works on linearscale envelope data.

$$
\begin{gathered}
\text { input }=\alpha+j \beta \\
\text { envelope }=a b s(\text { input })=|\alpha+j \beta|=\sqrt{\alpha^{2}+\beta^{2}}
\end{gathered}
$$


The data is normalized to the maximum output of the scanner and logarithmically compressed to a dynamic range of $60 \mathrm{~dB}$.

$$
\begin{gathered}
\text { data } \text { normalized }=\frac{\text { envelope }}{\max } \\
\log _{\text {_compressed }}=20 \cdot \log _{10}\left(\text { data }_{\text {normalized }}+10^{\frac{-60}{20}}\right)
\end{gathered}
$$

2) Segmentation: The first frame of logarithmically compressed data is subdivided into a number of segments. The segments are used to map deviations such as anechoic or hyper echoic areas in the ultrasound image. The segment size was chosen to be $0.81 \mathrm{~mm}$ in depth and $0.94^{\circ}$ in width. This resulted in 208 segments along the depth and 64 along the width, when using a scanning depth of $16.91 \mathrm{~cm}$ and a concave transducer with a sector angle of $60^{\circ}$. This ensures that individual speckles are not marked as a deviations and tissue transitions can be precisely defined.

3) Mapping: The average grayscale intensity of each segment is found and compared to the average grayscale intensity of the entire frame. The segment is mapped as bright if the intensity is greater than $111 \%$ of the intensity of the entire frame and dark if the intensity is less than $91 \%$ of the intensity of the entire frame. A mask is created wherein the deviating segments are mapped according to whether they are dark or bright (see Table Ia). In the mask, a further differentiation between deviations is conducted. If a given row contains more than $80 \%$ bright segments, the entire row is mapped as a near field hyper intensity areas (NFHIA). Equivalently, if a row contains more than $80 \%$ dark segments, the entire row is mapped as a deep area of low signal strength (DALSS). All thresholds were found empirically. The mask is now a map of the frame containing the locations of anechoic areas such as cysts and hyper echoic areas such as vessel walls (see Table $\mathrm{Ib})$.

4) Compensation: In finding an estimate of the attenuation of sound waves the average power of the logarithmically compressed data is calculated, such that a curve of average power is found along the depth axis of the frame. This average power curve is much affected by the demarcated deviating areas. The influence of these areas is therefore limited. By using the mask, the deviating areas are found in the logarithmically compressed data, and here they are assigned the average intensity of the entire frame (see Table Ic). To create a more uniform gray level appearance a 3rd order polynomial is fitted to the logarithmically compressed average power curve instead of a straight line as done by Lee et al. [1]. By subtracting the 3rd order polynomial from the mean of the average power the coefficients needed for compensating each row of data was found (see Fig. 1). The found coefficients are then converted to linear scale and multiplied onto each row of the unprocessed frame. Each frame of a data set can be processed individually or the calculated compensation can be applied to several frames, thereby saving processing time.

\section{B. Data acquisition}

An experienced sonographer (MMP) scanned the abdomen of two healthy volunteers and 40 ultrasound sequences were
TABLE I

PSEUDO-CODE
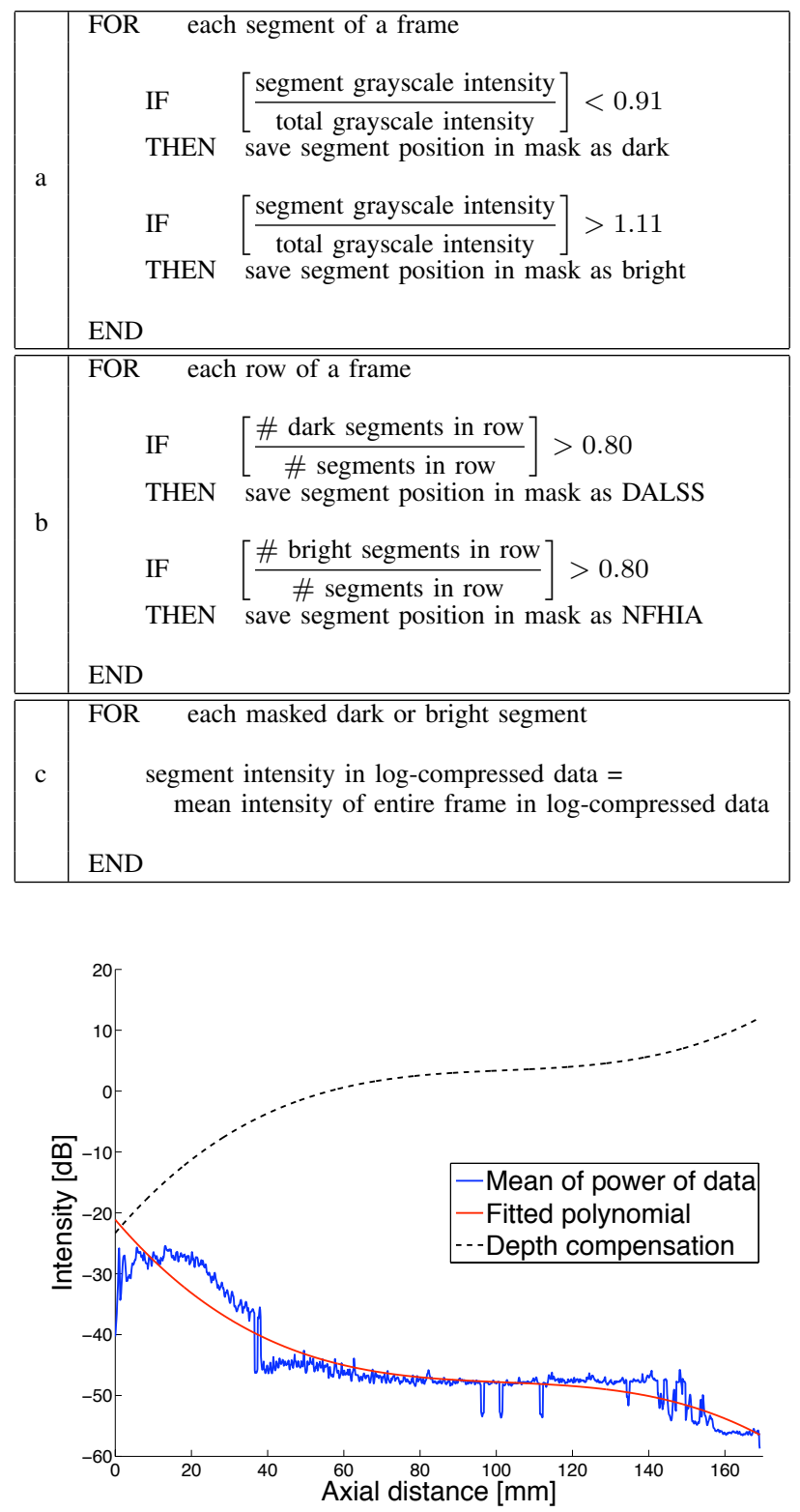

Fig. 1. The bold lines are the average power of a frame (blue) and the corresponding fitted 3rd order polynomial (red). The dashed 3rd order polynomial is the depth compensation (black).

recorded. The length of each sequence was 5 seconds and the frame rate was 40 frames per second. For recording the data a commercial ultrasound scanner (2202 ProFocus, BK Medical, Denmark) with a 192 elements concave transducer (8820e BK Medical) was used. The TGC was set at an all center position, ensuring that all sequences were recorded with the same standard gain. Beamformed in-phase and quadrutare phase (IQ) data were acquired using a research interface connected to the scanner [3]. This interface was also used to verify the centering of the TGC. 


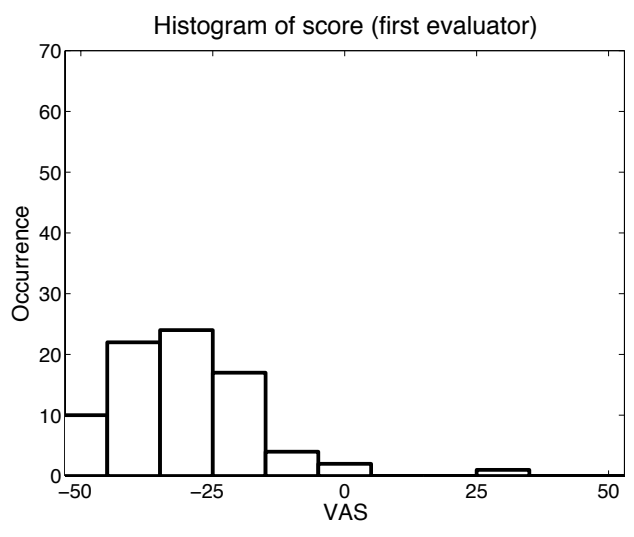

(a)

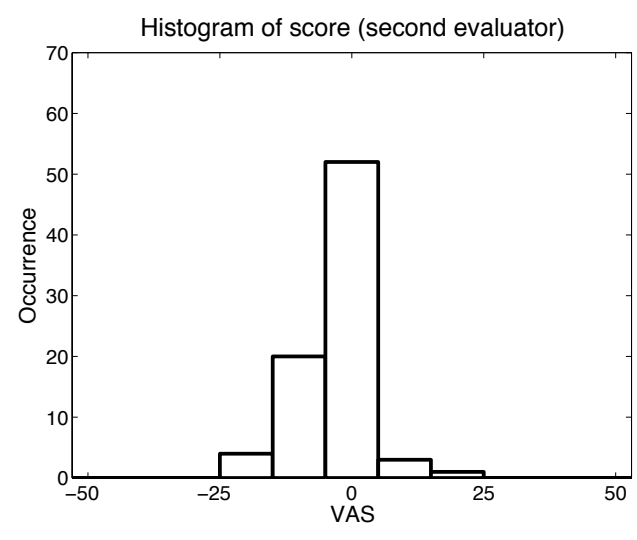

(b)

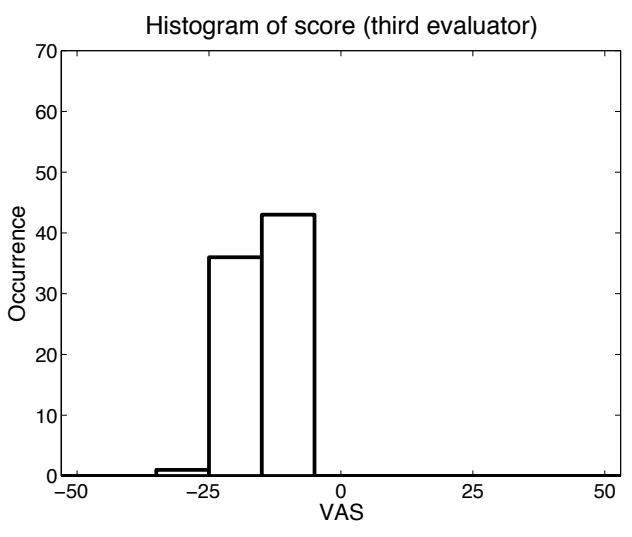

(c)

Fig. 2. Histograms of three evaluators VAS

\section{Evaluation of algorithm}

To get a measure of the performance of the developed algorithm, five ultrasound experts evaluated the recorded movie sequences (see Section II-B Data acqusition). First they evaluated the image quality, and then the penetration depth. Of the five experts, one recorded the ultrasound sequences two weeks prior to the evaluation. He was aware that the project concerned ATGC, but was not involved in the development of the algorithm. He had not seen the processed sequences prior to the evaluation. The remaining four experts were not involved in the project and had no prior knowledge of the developed algorithm or the resulting images. Evaluations were done blinded and independently of each other one expert at the time, using the program IQap [3]. Evaluations were conducted in a darkened room without windows. No rescaling or image compression was used.

The image quality was evaluated by displaying matching pairs of processed and unprocessed movie sequences side-byside. Discontinuities was avoided by playing each movie sequence continuously forwards and backwards. The evaluation was double blinded by random left right placement of the processed and unprocessed sequences and by randomizing the order of which the sequence pairs were displayed. Furthermore each sequence pair was displayed twice with different permutations. This resulted in 80 presentations of the 40 pairs. The experts scored the image quality of each pair on a visual analog scale (VAS) by dragging a marker towards the preferred movie sequence according to the steps shown in Fig. 3. The experts were asked to compare the sequences on their relative clinical value.

In the evaluation of the penetration depth, the movie sequences were displayed to the expert one at the time. The expert was asked to place a red bar to the depth after which the image quality had no clinical value. This was performed for each processed and unprocessed movie sequence, giving a total of 80 penetration depth evaluations per expert.

\section{RESUlTS AND DiscuSSION}

The developed algorithm generally improves image quality and penetration depth of the recorded in-vivo ultrasound sequences. An example is shown in Fig. 4.

\section{A. Statistical analysis of image quality}

The statistical analysis of the image quality consist of, first a consistency check, and then a test of the actual image quality. When testing for consistency, a Wilcoxon paired signed-rank test is used to see whether the experts scored the sequences with a statistically significant difference according to their leftright placement. Here two of the five experts were found to be inconsistent at a $5 \%$ level of significance.

A Wilcoxon signed-rank test was used to analyze the results from the image quality evaluation. Due to subjective interpretations of the VAS, it is not possible to compare the scores of the experts with each other. The test shows a statistically significant favoring of the processed sequences, without regard to consistency, with $\mathrm{P}$-values in the order of $10^{-8}-10^{-14}$. The scores from the three experts that were not inconsistent are shown in Fig. 2. The result displayed in Fig. 2a is from the expert that recorded the evaluated sequences. This evaluation may have been affected by the expert's involvement in the process. A further investigation of the effect of the involvement is not possible due to the subjective interpretations of the VAS, but since the P-value is equally small for all experts, the results are included.

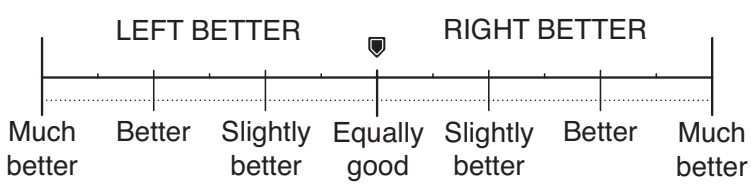

Fig. 3. Visual analog scale (VAS) 


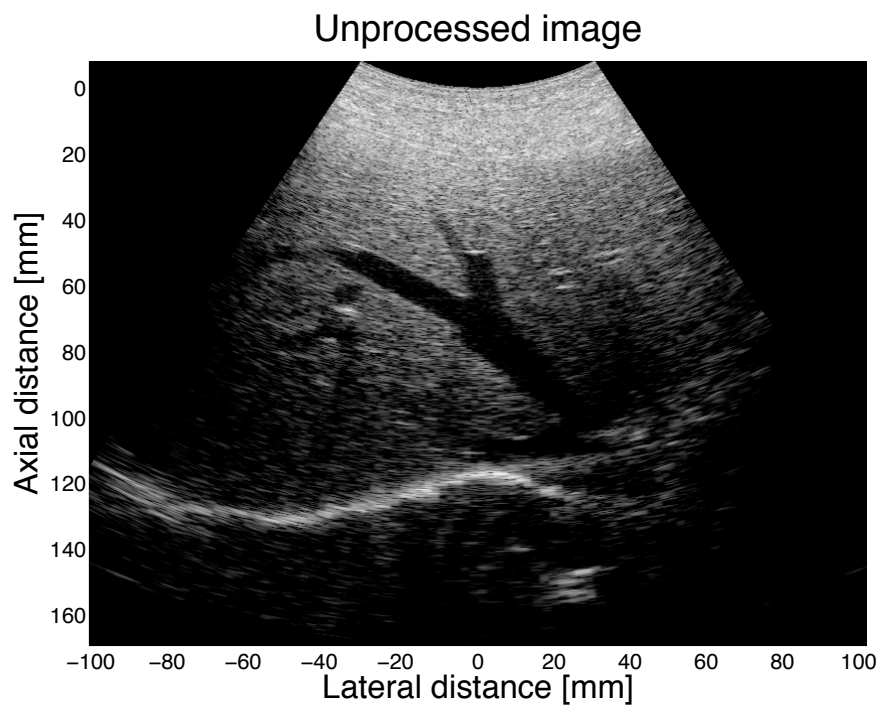

(a)

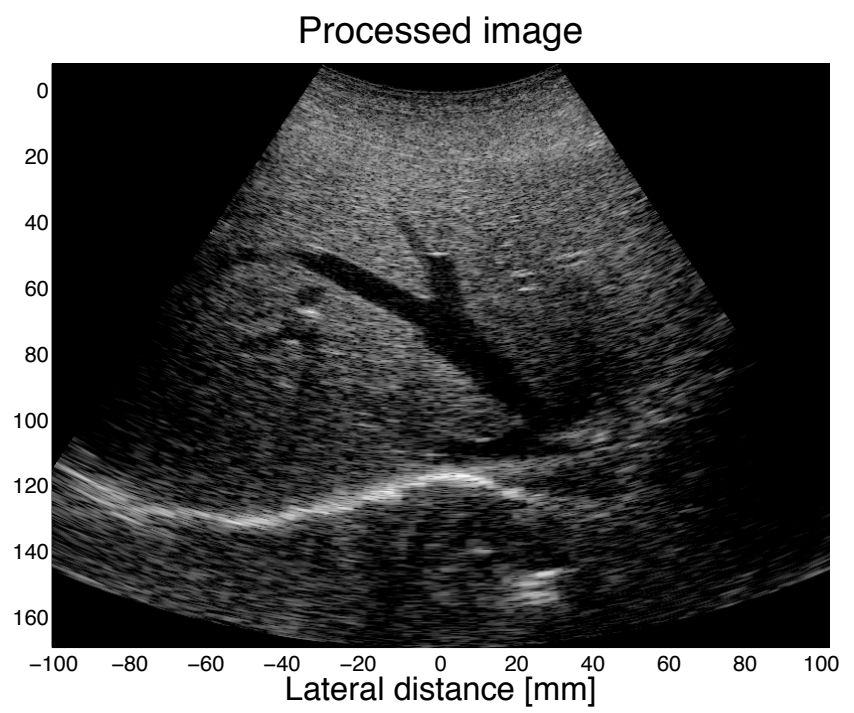

(b)

Fig. 4. Unprocessed (a) and processed (b) image

\section{B. Statistical analysis of penetration depth}

Testing the results with a Wilcoxon paired signed-rank test, the results shown in Table II are achieved. Here low p-values are seen among 4 out of the 5 experts. Considering the pvalues, experts 1,4 and 5 show substantial increase in depth from the unprocessed to the processed sequences. Expert 1 is the sonographer that recorded the ultrasound sequences. When looking at the p-values in Table II, the results from expert 1, however, does not stand out. Experts 2 and 3 are significantly different from the remaining three experts and do not show a significant increase in penetration depth at a $5 \%$ level of significance. The reason for this may be found in the given evaluation criterion. If the position of the diaphragm is chosen as the penetration depth in the unprocessed sequences, it will most likely also be chosen as the penetration depth in the processed sequences, as the diaphragm is seen as a saturated line. The visibility of the diaphragm can be clinically relevant in some situations, and it will often be visible on abdominal ultrasound scans even though details just above the diaphragm are missing. The effect of the ATGC will thereby not be rated. If considering the pooled data, a statistically significant increase of $1.91 \mathrm{~cm}$ is seen from the unprocessed

TABLE II

STATISTICAL RESULTS OF PENETRATION DEPTH ASSESSMENT

\begin{tabular}{lcc}
\hline Expert & $\begin{array}{c}\text { Mean difference } \\
\text { in depth }[\mathrm{cm}]\end{array}$ & P-value \\
\hline 1 & 3.47 & $1.20 \cdot 10^{-8}$ \\
2 & 0.40 & 0.068 \\
3 & 0.68 & 0.395 \\
4 & 2.49 & $5.72 \cdot 10^{-8}$ \\
5 & 2.48 & $1.00 \cdot 10^{-10}$ \\
pooled & 1.91 & $1.19 \cdot 10^{-18}$ \\
\hline
\end{tabular}

to the processed sequences. Figure 4 shows a sequence frame processed by the algorithm (right) and the corresponding unprocessed frame (left).

\section{CONCLUSION}

A new algorithm for ATGC has been developed and clinically evaluated. With P-values from $10^{-8}-10^{-14}$ in the image quality evaluation and a pooled P-value of $10^{-18}$ for the penetration depth evaluation, it is possible to state that the algorithm is capable of creating a uniform gray-level appearance, and of compensating for the depth attenuation on abdominal in-vivo ultrasound images.

\section{ACKNOWLEDGMENT}

Thanks to the experts and the volunteers for participating. Thanks to BK Medical Aps for providing an ultrasound scanner making in-vivo recording possibly. Thanks to Andreas Kamp Bünger for graphical assistance.

\section{REFERENCES}

[1] D. Lee, Y. S. Kim and J. B. Ra, Automatic time gain compensation and dynamic range control in ultrasound imaging systems, SPIE Medical Imaging, vol. 6147, pp. 614708-1 - 614708-9, 2006.

[2] S. D. Pye, S. R. Wild and W. N. McDicken, Adaptive time gain compensation for ultrasonic imaging, Ultrasound in Med. \& Biol., vol 18, no. 2, pp. 205-212, 1992.

[3] M. C. Hemmsen, M. M. Pedersen, S. I. Nikolov, M. B. Nielsen, and J. A. Jensen, Ultrasound Image Quality Assessment: A framework for evaluation of clinical image quality, SPIE Medical Imaging, vol. 7629, pp. 76290C-1 - 76290C-12, 2010.

[4] S. D. Pye, S. R. Wild and W. N. McDicken, Clinical trial of a new adaptive TGC system for ultrasound imaging, The British Journal of Radiology, 61, pp. 523-526, 1988.

[5] D. I. Hughes and F. A. Duck, Automatic attenuation compensation for ultrasonic imaging, Ultrasound in Med. \& Biol., vol. 23, no. 5, pp. 651664, 1997. 